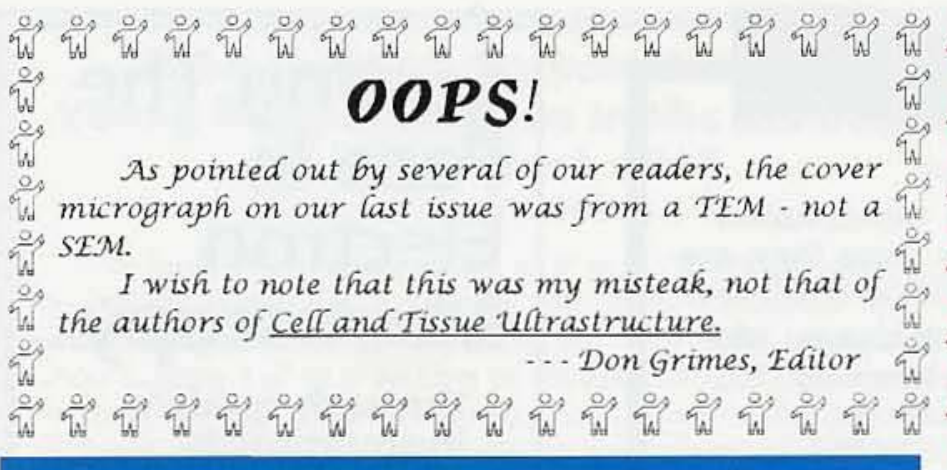

\section{Biology For Nonbiologists And Support People} Sterling P. Newberry

The fact that about half of our readers are not biologists does not mean that they are disinterested in the fascinating current developments in the field of biology any more than the biologist are disinterested in the equally fascinating progress of materials science or physics. Popular press and even trade journals often over simplify or at best give small pieces of information. It is nice when a refereed journal gives a state of the art review. Science Magazine recently gave such a review under the title "Development Frontiers in Biology"'. As is customary with Science, this issue also contains editorial summaries of many of the contributions in less technically obscure language.

I highly recommend this issue to all our readers. One should note that some of the new findings would have been very difficult or impossible without the use of microscopy and that the unsolved problems which are listed will need analytical microscopy to move hand in hand with the genetic testing methods which are becoming so powerful.

1. Science vol. 266 , pps. 561 to 614, Oct. 28 (1994)

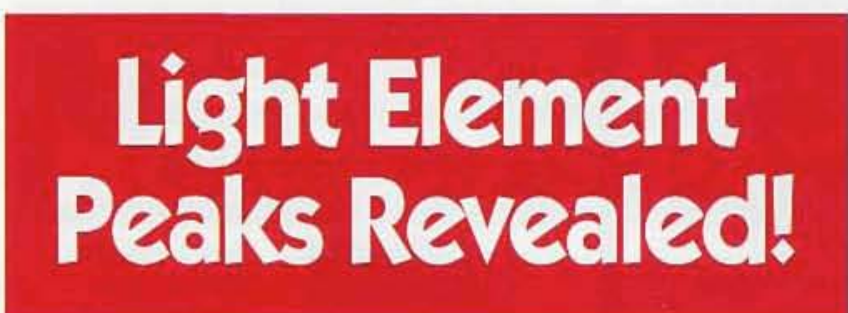

\section{Oil Film on EDX Windows Removed:}

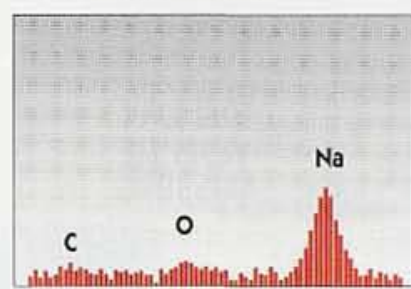

Oily Window

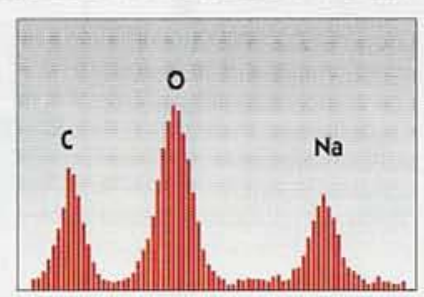

Clean Window
Oil build-up on EDX detector windows can ruin sensitivity for light element X-rays in SEMs. To stop oil condensation and keep the system clean, smart SEM users rely on the XEI Scientific SEM-CLEAN ${ }^{\mathrm{TM}}$ system.

Result: Consistent light element X-ray results and contaminationfree pictures. The Nitrogen purge of the inexpensive SEM-CLEAN system actively cleans your electron microscope while you're away.

\section{SEM-CLEAN'" Stops the Oil

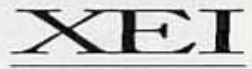 \\ S CIENTIFIC}

3124 Wessex Way, Redwood City, CA 94061 415-369-0133 - Fax 415-363-1659

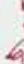

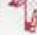

tip

(1)

$\frac{t}{2}$

4.

to.

to.

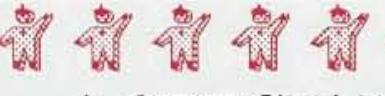

\section{A SPECIAL THANKS!!!}

The objective of this newsletter remains, perhaps unlike many others, to publish material and informaion of interest to the working microscopist.

And, as advertising thankfully increases, so does he challenge of obtaining an appropriate proportion of material and information. With this challenge in mind, I would like to sincerely thank the following individuals who have made contributions to the newsletter during he past year:

\section{Gib Ahlstand, Univ. of Minnesota} Jessica Bailey, Handmade Software, Inc. John Bozzola, Southern Illinois University Charles E. Bryson III. Surface/Interface, Inc. Anthony D. Buonaquisit, AMT Stephen W. Carmichael, Mayo Clinic

Don Chernoff, Small World

A. Kent Christensen. Univ. of MI Medical School Locke Christman, FEI Company

Briggs Christie, TopoMetrix

L.S. Chumbley, Iowa State Univ.

Theodore M. Clark. J.I. Case

George J. Collins, TopoMetrix

Robert Compton, Consultant

K. Cooper, McMaster Univ. Medical Cur Donald P. Cox, Goldmark Biologicals

N.D. Evans, Oak Ridge Institute

Barbara Foster, MM\&E

David P. Field, TexSem Laboratories

Jacqueline Gallet, Univ. of California Irvine

Monte G. Heaton, Digital Instruments

Damon Heer, FEI Company

Cindy Henk, Louisiana State Univ.

Kevin M. Imel, Washington State Univ.

Donald R. Laferty, Jr., Leica, Inc.

Cindy L. Lewis, Children's Hosptial Med Ctr

Mark W. Lund, MOXTEK

Charles Lyman. Lehigh Universtiy

John Mansfield, University of Michigan

Barry D. Masters, Uniformed Services Univ

Kevin McCarthy, University of Alabama

Walter C. McCrone, McCrone Research Inst

Judy Murphy, San Joaquin Delta College

Sterling P. Newberry, Consultant

Leonard Polizzotto, Polaroid Corporation

M.T. Postek, NIST, Gaithersburg

Jean-Paul Revel, CALTECH

Mark Rigler, Materials Analytical Services

T.G. Rochow, North Carolina State Univ.

Krystyna Rybicka, SUNY at Buffalo

Jan S. Ryerse, St Louis Univ Health Sci Ctr

Alan Sandborg, EDAX International

M. Eric Schlienger, Virtual Laboratories

Caroline Schooley. Consultant

Supapan Seraphin, Univ. of Arizona

Farhad Shaapur, Arizona State University

Kenneth Smith, McCrone Associates

Robert Summers, SUNY Buffalo

A.J. Terry, ORTECH Corporation

Scott D. Walck, Wright-Patterson AFB

Daphna R. Yaniv. Arizona State Univ.

Speaking of "thanks", should you find the publication of some value, you might thank your sales representative for the advertising support of his/her company. 

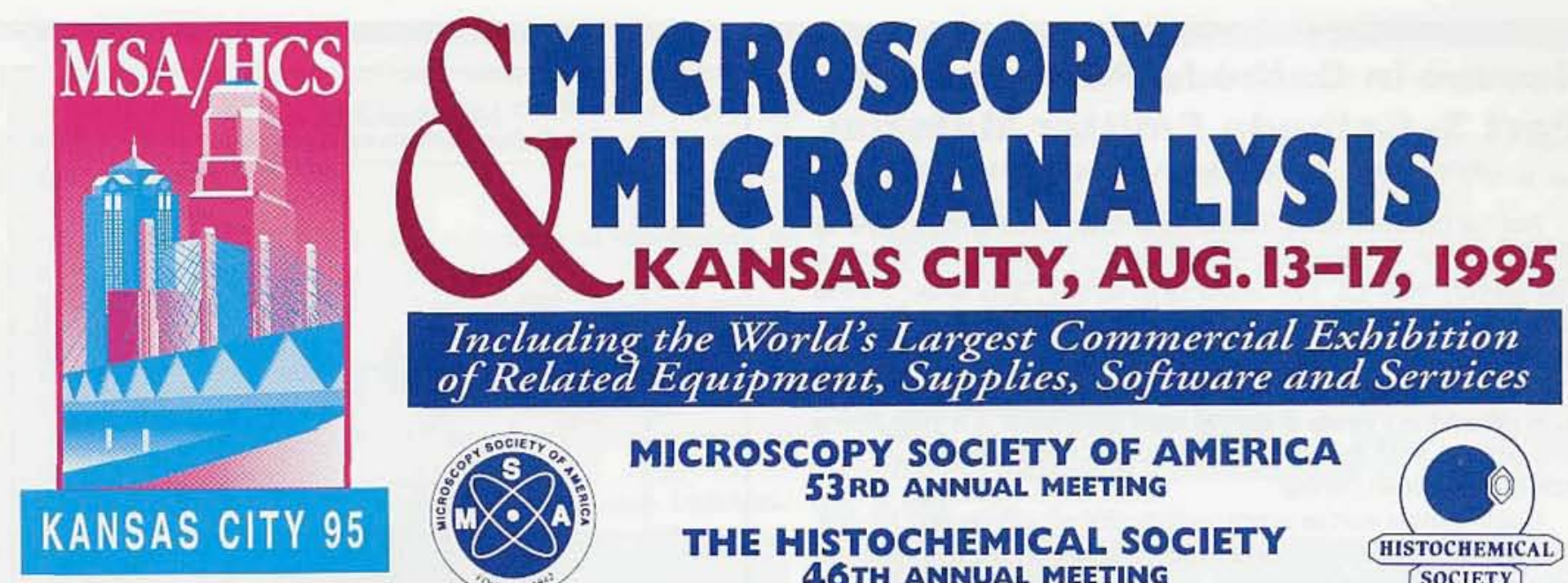

Including the World's Largest Commercial Exbibition of Related Equipment, Supplies, Software and Services

\section{SYMPOSIA AND CONTRIBUTED SESSIONS}

$\nabla$ Instrumentation \& Methodologies

3-D Imaging \& 3-D Reconstruction Advanced Computing in Microscopy Advances in Light Microscopy

Auger Electron Spectroscopy

Biomaterials

Confocal Microscopy

Convergent Beam Diffraction

Correlative Microscopy (Tech Forum)

Developments in Computer Controls

Direct Digital Imaging \& Microscope Automation

Energy Loss \& Filtering Spectroscopy

Field Emission

Image Formation \& Recording

Image Processing, Analysis, \& Modeling

Microbeam Mass Spectroscopy

Near Field Microscopy

Optical \& Electron Holography

Optical Microspectroscopies

Scanning Probe Microscopy \& Analysis

SEM \& TEM/STEM

Surface Analysis Methods

High-Res Intermediate- \& High-Voltage

EM in Biology \& Materials Science

Tomography

X-ray EDS \& XRF/XRD Techniques

\section{SHORT COURSES}

Immunocytochemistry \& Protein Labeling: Ag-Enhanced Small Au Particles

Approaches for in situ PCR \& in situ

Hybridization in Research \& Diagnosis

Cryotechniques for TEM, \& Low-

Temperature SEM

Electron Diffraction in the TEM

Introduction to Digital Imaging

\section{$\nabla$ Biological Sciences}

Applied Biomedical Microscopies

Cryotechniques

Cytochemistry/Histochemistry

EM Tomography in Biology

Extracellular Matrix/Cell-ECM Interactions

Functional Imaging of the Nervous System

Functional Organization of the Cell Nucleus

Histochemistry: Nucleic Acid Detection Immunology \& Immunocytochemistry In Situ Hybridization

Macromolecular Microscopy

Membranes, Microdomains \& Transport

Microbiology

MRI Microscopy

Organelles, Chromosomes \& Nuclei

Pathology

Phytomorphology \& Phytobiology

Reproduction \& Developmental Biology

Scanned Probe Microscopy

\section{IN ADDITION}

Over 130 Commercial Exhibits

Over 600 Scientific Papers

Over 50 Scientific Sessions

Computer Software Exchange

8th Annual Golf Tournament

Postage Stamp Exhibit

3rd Annual 5k Run/Walk

Best Poster \& Photomicrograph Awards

Educational Outreach Program

Public Education Panel

Histochemical Society Lillie Awards

MSA Presidential Student Awards

EM Facilities Management (Tech Forum)

\section{$\nabla$ Physical Sciences}

Amorphous/Disordered Materials Analytical Microscopy Advances: Ceramics Approaches to Catalyst Characterization Coatings

Electronic Materials

Geology/Mineralogy

High Spatial Resolution Microanalysis \& Microscopy

In Situ Microscopy in Materials Research Magnetic Materials

Microscopic Characterization of Materials Nano-Phase \& Nano-Composite Materials Oxidation \& Corrosion

Petrology

Phase Transformations

Polymers, Metals \& Alloys, Ceramics

Quantitative Electron Diffraction

Radiation-Sensitive Materials

Small Particles/Clusters

Surfaces \& Interfaces

Superconductors

Tribology

\section{TUTORIALS}

Exploring Local Properties of Biological

Surfaces with Atomic Force Microscopy

Histochemical Methods for Localizing \& Characterizing Glycoconjugates

Energy Filtering in Biological EM

Navigating the Internet

Digital Imaging

Electron Backscattered Diffraction: An Emerging Probe for Materials Characterization

Atom Probe Analysis of the Solid-Liquid Interface

Image Analysis Fundamentals

\begin{tabular}{c} 
FOR MORE INFORMATION \\
\hline Meeting Office $\nabla$ P.O. Box EM $\nabla$ Woods Hole, MA 02543 \\
Toll-Free: 800-538-3672 $\nabla$ Fax: 508-548-9053 $\nabla$ E-mail: Imaser@mbl.edu \\
\hline
\end{tabular}

